\title{
Total and Dietary Calcium Intake and Colorectal Adenoma in Korean Adults
}

\author{
Ju Eun Seol ${ }^{1}$, Chang Ho Cho ${ }^{2}$, Sung Hi Kim³ ${ }^{3}$ Jung Eun Lee ${ }^{1}$ \\ 'Department of Food and Nutrition, College of Human Ecology, Sookmyung Women's University, Seoul, ${ }^{2}$ Departments of Pathology and ${ }^{3}$ Family \\ Medicine, Daegu Catholic University Medical Center, Daegu, Korea
}

\begin{abstract}
Background: Colorectal cancer in Korea has become more prevalent over the few last decades, and calcium is considered a preventive factor for colorectal cancer development. We examined the associations between total and dietary calcium intake and the prevalence of colorectal adenoma in Korean adults.

Methods: This cross-sectional study included 112 colorectal adenoma cases and 252 adenoma-free non-cases, aged 45 to 71 years, who underwent colonoscopies at the Daegu Catholic University Medical Center from August 2011 to September 2012. Participants were asked about their diet using a validated food frequency questionnaire and about supplement use through interviews. We calculated odds ratios (ORs) and $95 \%$ confidence intervals (Cls) to evaluate the association between total and dietary calcium intake and the prevalence of colorectal adenomas using multivariable logistic regression models.

Results: Increasing total calcium intake from foods and supplements was significantly associated with a decreased prevalence of colorectal adenoma in women; comparing the highest quartile with the lowest quartile, the OR $(95 \% \mathrm{Cl})$ was $0.35(0.15-0.85 ; P$ for trend $=$ 0.03). Likewise, high dietary calcium intake from foods was associated with a lower prevalence of colorectal adenoma in women; compared with the lowest quartile, the ORs $(95 \% \mathrm{Cls})$ were $0.32(0.13-0.82)$ for the 3 rd quartile and $0.44(0.19-1.03 ; P$ for trend $=0.13)$ for the 4th quartile. However, the association was not clear for either total or dietary calcium intake among men.
\end{abstract}

Conclusions: A higher intake of calcium was associated with a reduction of colorectal adenoma prevalence in Korean women.

(J Cancer Prev 2015;20:153-158)

Key Words: Calcium, Colorectal neoplasms

\section{INTRODUCTION}

Colorectal cancer is the third leading cause of death from cancer worldwide, ${ }^{1}$ and the incidence rates in Korea has increased continuously by $5.3 \%$ annually from 1999 to $2012 .^{2}$ Colorectal adenoma is widely accepted as a precursor of colorectal cancer, which is the consequence of the adenoma-carcinoma sequence. ${ }^{3}$ In this respect, identifying the potential protective factors of colorectal adenoma may be necessary for proposing prevention strategies to reduce colorectal cancer.

Epidemiologic evidence suggests that calcium intake may prevent the development of colorectal adenoma or cancer. A recent meta-analysis of observational studies reported that calcium intake may help reduce the risk of colorectal adenomas, particularly high risk adenomas, ${ }^{4}$ and another meta-analysis of 60 observational studies also reported that both dietary and supplementary calcium intake reduced the risk of colorectal cancer. ${ }^{5}$ However, a large randomized double-blind clinical trial did not find any benefit of calcium supplementation on colorectal cancer. $^{6}$

Calcium may inhibit carcinogenesis through a direct suppression of cancer growth, cell differentiation and apoptosis. ${ }^{7}$ Although the role of calcium in the development of colorectal neoplasia has been largely explored in Western populations, there have been

Received June 1, 2015, Revised June 12, 2015, Accepted June 13, 2015

Correspondence to: Jung Eun Lee

Department of Food and Nutrition, College of Human Ecology, Sookmyung Women's University, 100 Cheongpa-ro 47-gil, Yongsan-gu, Seoul 140-742, Korea Tel: +82-2-2077-7560, Fax: +82-2-710-9479, E-mail: junglee@sm.ac.kr, ORCID: Jung Eun Lee, http://orcid.org/0000-0003-1141-878X

Copyright (C) 2015 Korean Society of Cancer Prevention

(c) This is an Open Access article distributed under the terms of the Creative Commons Attribution Non-Commercial License (http://creativecommons.org/licenses/by-nc/4.0) which permits unrestricted non-commercial use, distribution, and reproduction in any medium, provided the original work is properly cited. 
only a few studies on calcium intake and colorectal neoplasia in Asian populations. AJapan Public Health Center-based Prospective Study found an inverse association of dietary calcium intake with colorectal adenoma, ${ }^{8}$ and colorectal cancer, ${ }^{9}$ and another cohort study among Chinese women showed a reduction in the risk of colorectal cancer with high calcium intake. ${ }^{10}$

Given the limited evidence regarding an association between calcium intake and colorectal neoplasia in Korean populations, whose calcium intake is lower than that of Western populations, we aimed to evaluate the association between calcium intake and the prevalence of colorectal adenomas in a cross-sectional study of Korean adults.

\section{MATERIALS AND METHODS}

\section{Study population}

Our study included 112 colorectal adenoma cases and 252 adenoma-free non-cases, aged 45 to 71 years. The patients were consecutively admitted to the study at the Daegu Catholic University Medical Center in Daegu, Korea, from August 2011 to September 2012. Participants underwent a colonoscopy, and the histological features of the adenomas were examined by a pathologist. Participants were asked about their socio-demographic status, alcohol intake, smoking status, family history of colorectal cancer, and the use of supplements through structured questionnaires. Regarding supplements used, we also asked participants about the brand name, dose and duration. Dietary intake was assessed using a validated food frequency questionnaire. ${ }^{11}$ Dietary calcium intake from foods was calculated by multiplying the reported frequency of each relevant food by the nutrient content of one serving of that food. Total dietary calcium was calculated by summing dietary calcium intake and calcium intake from supplements. Weight and height were directly measured and body mass index (BMI, $\mathrm{kg} / \mathrm{m}^{2}$ ) was calculated by dividing the weight in kilograms by the square of the height in meters. We excluded those with history of cancer $(n=14)$ or unreasonable energy intake (beyond the mean of the logarithm of energy \pm 3 standard deviation [SD]) $(n=4)$. This study was approved by the Institutional Review Board of Daegu Catholic University Medical Center, and written informed consent was obtained from all participants.

\section{Ascertainment of colorectal adenoma}

Colorectal adenomas were determined through colonoscopies and pathological examination. Polyps were classified as adenomatous, hyperplastic, or other non-adenomatous and we included adenomatous polyps as cases.

\section{Statistical analysis}

Baseline characteristics were compared between cases and non-cases using the means and SDs for continuous variables or the percentages for categorical variables. We used a t-test for continuous variables, and a chi-square test for categorical variables. Total and dietary calcium intakes were adjusted for energy by residual methods. ${ }^{12}$

We calculated the odds ratios (OR) and 95\% confidence intervals (CI) to evaluate the associations using the multivariable logistic regression models. Tests for trends were performed by treating the median value of each calcium quartile in the control group as a continuous variable in the models. We adjusted for age (years, continuous), sex (men or women), total energy intake (kcal/d, continuous), pack years of smoking (continuous), alcohol intake ( $\mathrm{g} / \mathrm{d}$, continuous), and education level (less than high school or high school graduate or above). We also examined the joint association of calcium intake with colorectal adenoma prevalence by BMI and smoking status and performed tests for interactions using a likelihood ratio test; BMI was divided into two categories ( $<23$ or $\geq 23 \mathrm{~kg} / \mathrm{m}^{2}$ ), and smoking status was also divided into two categories (never or ever smokers). All analyses were performed using SAS 9.3 (SAS Institute Inc., Cary, NC, USA). All statistical tests were two-sided, and $P$-values $<0.05$ were considered statistically significant.

\section{RESULTS}

Table 1 shows the baseline characteristics of the study population according to colorectal adenoma case status. The average ages were 60.3 years for cases, and 59.2 years for non-cases. The colorectal adenoma cases patients were more likely to be male, smoke, and drink alcohol compared to non-cases. We found that the total calcium intake from supplements and food was inversely associated with the prevalence of colorectal adenoma (Table 2); comparing the highest quartile with the lowest quartile, the OR ( $95 \% \mathrm{CI}$ ) was 0.49 (0.24-0.97; $P$ for trend = $0.04)$. This inverse association was mainly driven by the association in women for whom the OR $(95 \% \mathrm{CI})$ was 0.35 (0.15-0.85; $P$ for trend $=0.03$ ) when comparing the highest quartile with the lowest quartile. We did not find a significant association for men. Likewise, we found a lower prevalence of colorectal adenoma with high dietary calcium intake from foods in women (Table 3); compared with the lowest quartile, the ORs ( $95 \% \mathrm{CIs}$ ) were 0.32 (0.13-0.82) for the 3rd quartile and 0.44 (0.19-1.03; $P$ for trend $=$ 
Table 1. Characteristics of patients according to colorectal adenoma case status

\begin{tabular}{|c|c|c|c|}
\hline Variable & Adenoma $(\mathrm{n}=112)$ & No adenoma $(\mathrm{n}=252)$ & $P$-value ${ }^{\mathrm{a}}$ \\
\hline Age $(y r)$ & $60.3 \pm 5.3$ & $59.2 \pm 5.0$ & 0.44 \\
\hline Sex & & & $<0.0001$ \\
\hline Men & $58(51.8)$ & $69(27.4)$ & \\
\hline Women & $54(48.2)$ & $183(72.6)$ & \\
\hline Body mass index $\left(\mathrm{kg} / \mathrm{m}^{2}\right)$ & & & 0.21 \\
\hline$<23$ & $33(29.5)$ & $73(29.0)$ & \\
\hline $23-25$ & $32(28.6)$ & $94(37.3)$ & \\
\hline$\geq 25$ & $47(42.0)$ & $85(33.7)$ & \\
\hline Education & & & 0.12 \\
\hline Less than high school graduate & $41(36.6)$ & $114(45.2)$ & \\
\hline High school or above & $71(63.4)$ & $138(54.8)$ & \\
\hline Pack years of smoking & $8.8 \pm 13.1$ & $4.5 \pm 11.2$ & 0.05 \\
\hline Alcohol intake $(g / d)$ & $16.6 \pm 25.2$ & $6.5 \pm 18.8$ & 0.0002 \\
\hline Family history of colorectal cancer & & & 0.32 \\
\hline No & $106(94.6)$ & $244(96.8)$ & \\
\hline Yes & $6(5.4)$ & $8(3.2)$ & \\
\hline Use of supplement containing calcium & & & 0.02 \\
\hline No & $92(82.1)$ & $179(71.0)$ & \\
\hline Yes & $20(17.9)$ & $73(29.0)$ & \\
\hline Dietary calcium intake from foods $(\mathrm{mg} / \mathrm{d})$ & $374.4 \pm 223.0$ & $435.2 \pm 331.7$ & $<0.0001$ \\
\hline Total calcium intake from supplements and foods $(\mathrm{mg} / \mathrm{d})$ & $407.8 \pm 237.6$ & $519.9 \pm 386.9$ & $<0.0001$ \\
\hline
\end{tabular}

Values are presented as mean \pm standard deviation or number (\%). ${ }^{a} t$-test for continuous variables and chi-square test for categorical variables.

Table 2. Odds ratios (ORs) and $95 \%$ confidence intervals ( $95 \% \mathrm{CIs}$ ) for adenoma status according to total calcium intake from foods and supplements

\begin{tabular}{lcccc}
\hline & \multicolumn{4}{c}{ Total calcium intake } \\
\cline { 2 - 5 } & Quartile 1 & Quartile 2 & Quartile 3 & Quartile 4 \\
\hline All patients & & & & \\
Median (mg/d) & 211.27 & 364.22 & 502.69 & 807.94 \\
Case/control (n) & $38 / 53$ & $31 / 60$ & $24 / 67$ & $19 / 72$ \\
OR (95\% CI) & 1.00 & $0.72(0.39-1.36)$ & $0.60(0.31-1.16)$ & $0.49(0.24-0.97)$ \\
Women & & & & 0.04 \\
Median (mg/d) & 208.33 & 361.44 & 504.84 & $12 / 61$ \\
Case/control (n) & $16 / 29$ & $13 / 39$ & $13 / 54$ & $0.35(0.15-0.85)$ \\
OR (95\% CI) & 1.00 & $0.60(0.25-1.45)$ & $0.45(0.19-1.07)$ & 0.03 \\
Men (n) & & & & 712.69 \\
Median (mg/d) & 216.53 & 370.94 & 488.94 & $7 / 11$ \\
Case/control & $22 / 24$ & $18 / 21$ & $11 / 13$ & $0.81(0.26-2.58)$ \\
OR (95\% CI) & 1.00 & $0.84(0.35-2.06)$ & $0.79(0.28-2.23)$ & 0.67 \\
\hline
\end{tabular}

${ }^{a}$ Models were adjusted for the following covariates; age (years, continuous), sex, total energy (kcal/d, continuous), pack years of smoking (continuous), alcohol intake ( $\mathrm{g} / \mathrm{d}$, continuous), and education level (less than high school, more than high school). ${ }^{b}$ Models were adjusted for age (years, continuous), total energy (kcal/d, continuous), pack years of smoking (continuous), alcohol intake ( $\mathrm{g} / \mathrm{d}$, continuous), and education level (less than high school or high school graduate or above).

0.13) for the 4th quartile. However, there was a less clear association among men than among women. We examined the association between supplements containing calcium and colorectal adenoma prevalence. The ORs ( $95 \% \mathrm{CIs}$ ) comparing calcium-containing supplement use with non-use were 0.95 $(0.38-2.34)$ for men and $0.43(0.20-0.96)$ for women (data not shown). When we examined whether the associations varied by BMI, the association was more apparent among individuals with high BMI ( $\left.\geq 23 \mathrm{~kg} / \mathrm{m}^{2}\right)$ than those with low BMI $\left(<23 \mathrm{~kg} / \mathrm{m}^{2}\right)$, albeit not statistically significant (Table 4). We found evidence of a potential interaction with smoking status regarding the association between total calcium intake and colorectal adenoma. 
Table 3. Odds ratios (ORs) and $95 \%$ confidence intervals ( $95 \% \mathrm{CIs}$ ) for colorectal adenoma according to dietary calcium intake from foods

\begin{tabular}{lcccc}
\hline & \multicolumn{2}{c}{ Dietary calcium intake } \\
\cline { 2 - 5 } & Quartile 1 & Quartile 2 & Quartile 3 & Quartile 4 \\
\hline All patients & & & & \\
Median (mg/d) & 204.96 & 326.87 & 434.42 & 613.54 \\
Case/control (n) & $39 / 52$ & $28 / 63$ & $22 / 69$ & $23 / 68$ \\
OR (95\% CI) & 1.00 & $0.62(0.33-1.18)$ & $0.51(0.26-0.99)$ & $0.60(0.30-1.18)$ \\
Women & 195.77 & & & 014.64 \\
Median (mg/d) & $16 / 26$ & 326.87 & 439.06 & $16 / 60$ \\
Case/control (n) & 1.00 & $0.45(0.18-1.11)$ & $0.32(0.13-0.82)$ & $0.44(0.19-1.03)$ \\
OR (95\% CI) & & & & 0.12 \\
Men & 211.27 & 326.69 & 418.37 & 590.53 \\
Median (mg/d) & $23 / 26$ & $16 / 18$ & $12 / 17$ & $7 / 8$ \\
Case/control (n) & 1.00 & $0.80(0.31-2.03)$ & $0.71(0.27-1.90)$ & $0.90(0.27-3.03)$ \\
OR (95\% CI) & & & & 0.70 \\
\hline
\end{tabular}

${ }^{a}$ Models were adjusted for the following covariates; age (years, continuous), sex, total energy (kcal/d, continuous), pack years of smoking (continuous), alcohol intake ( $\mathrm{g} / \mathrm{d}$, continuous), and education level (less than high school, more than high school). ${ }^{\mathrm{b}}$ Models were adjusted for age (years, continuous), total energy (kcal/d, continuous), pack years of smoking (continuous), alcohol intake (g/d, continuous), and education level (less than high school or high school graduate or above).

Table 4. Odds ratios (ORs) ${ }^{a}$ and $95 \%$ confidence intervals $(95 \%$ CIs) for colorectal adenoma according to total calcium intake by BMI or smoking status

\begin{tabular}{|c|c|c|c|c|c|}
\hline & \multicolumn{4}{|c|}{ Total calcium intake } & \multirow{2}{*}{$\begin{array}{c}P \text { for } \\
\text { interaction }\end{array}$} \\
\hline & Quartile 1 & Quartile 2 & Quartile 3 & Quartile 4 & \\
\hline \multicolumn{6}{|c|}{ Body mass index $\left(\mathrm{kg} / \mathrm{m}^{2}\right)^{\mathrm{a}}$} \\
\hline Case/non-case (n) & $8 / 13$ & $7 / 20$ & $9 / 18$ & $9 / 22$ & \\
\hline$<23^{\mathrm{c}}$ & 1.00 & $0.33(0.08-1.39)$ & $0.49(0.12-1.99)$ & $0.67(0.18-2.43)$ & \\
\hline Case/non-case (n) & $30 / 40$ & $24 / 40$ & $15 / 49$ & $10 / 50$ & \\
\hline$\geq 23^{c}$ & 1.00 & $0.81(0.40-1.66)$ & $0.51 \quad(0.23-1.12)$ & $0.35(0.15-0.84)$ & 0.24 \\
\hline \multicolumn{6}{|l|}{ Smoking status ${ }^{\mathrm{b}}$} \\
\hline Case/non-case (n) & $24 / 35$ & $13 / 45$ & $14 / 57$ & $11 / 66$ & \\
\hline Never ${ }^{c}$ & 1.00 & $0.42(0.19-0.95)$ & $0.39(0.18-0.88)$ & $0.26(0.11-0.61)$ & \\
\hline Case/non-case (n) & $14 / 18$ & $18 / 15$ & $10 / 10$ & $8 / 6$ & \\
\hline Ever $^{c}$ & 1.00 & $1.46(0.53-4.05)$ & $1.13(0.34-3.74)$ & $1.80(0.48-6.72)$ & 0.03 \\
\hline
\end{tabular}

${ }^{a}$ Models were adjusted for the following covariates; age (years, continuous), sex, total energy (kcal/d, continuous), pack years of smoking (continuous), alcohol intake ( $\mathrm{g} / \mathrm{d}$, continuous), and education level (less than high school, more than high school). ${ }^{b}$ Models were adjusted for the following covariates; age (years, continuous), sex, total energy ( $\mathrm{kcal} / \mathrm{d}$, continuous), alcohol intake ( $\mathrm{g} / \mathrm{d}$, continuous), and education level (less than high school or high school graduate or above). 'OR (95\% CI).

Among never smokers, the ORs (95\% CIs) comparing the lowest quartile were $0.39(0.18-0.88)$ for the 3rd quartile and 0.26 $(0.11-0.61)$ for the 4 th quartile ( $P$ for interaction $=0.03$ ). However, we did not find a statistically significant association among ever smokers. Because never smokers were more likely to be women, we examined the association only among male never smokers $(n=39)$; the OR $(95 \% \mathrm{CI})$ for comparing high with low categories (divided by median levels) was 0.46 (0.09-2.32) (data not shown).

\section{DISCUSSION}

In our cross-sectional study of 112 colorectal adenoma cases and 252 non-cases, we found that increasing total calcium intake was associated with a decreasing prevalence of colorectal adenomas in Korean women. However, we did not find a clear association for men. The more apparent association among never smokers in our study could suggest that the lack of association observed in men may be partly due to residual confounding by smoking or an interaction with smoking. Given that $69 \%$ of men were past or current smokers in our study, our small sample size 
did not allow us to further explore whether the presence of a significant association among women, but not among men is because of the sex difference or an interaction with smoking among men. This topic warrants further large-scale studies. We found a clearer association for total calcium intake from supplements and foods than for dietary calcium intake from foods in women. This finding suggests that calcium supplementation may also be associated with a lower prevalence of colorectal adenoma. We found a suggestive inverse association between the use of calcium-containing supplements and colorectal adenoma prevalence in women.

Our results were consistent with previous studies in which inverse associations between calcium intake and colorectal adenomas were also observed. In a randomized double-blind trial of calcium supplementation, ${ }^{13} 3 \mathrm{~g}$ of calcium carbonate $(1,200 \mathrm{mg}$ of elemental calcium) was found to prevent colorectal adenoma recurrence; the $\mathrm{OR}$ for any recurrence of adenoma with calcium compared with placebo was 0.85 ( $95 \% \mathrm{CI}=0.74$ to 0.98 ). Several observational studies also found a benefit of calcium intake on colorectal adenoma. ${ }^{4}$ In a Japanese cohort study, dietary calcium intake showed a non-linear inverse association with the lowest association for the 2nd quintile, but no further decline in the upper quintiles. ${ }^{8}$ In observational studies of Western populations, there is a high correlation between vitamin $\mathrm{D}$ and calcium intake because dairy products are major sources of the two nutrients and are often fortified with vitamin D and calcium. Therefore, in many cases, it may be difficult to disentangle the effect of dietary calcium intake from that of dietary vitamin D intake. However, although dairy products are still a major food source for calcium intake in Korean adults, vegetables are the top food source for calcium in Korea. ${ }^{14}$ Therefore, consistent findings from our study and other studies suggest that calcium per se may be a factor related to colorectal adenoma.

The potential mechanisms by which calcium may reduce the risk of colorectal neoplasia are as follows: 1) calcium binds toxic bile acids and fatty acids, which promote cytotoxic and mutagenic damage to colorectal epithelium ${ }^{15}$; and/or 2) calcium directly affects the cell cycle through calcium binding with the calcium sensing receptor. ${ }^{16,17}$

Our study has several strengths and limitations. To our knowledge, our study is the first to examine the association between calcium intake and colorectal adenoma in Korean populations. We investigated supplemental use in each individual and calculated calcium intake from supplements and were therefore able to examine both total and dietary calcium intake in relation to colorectal adenoma. We adjusted for potential confounding factors. However, the limitations of our study include a small sample size, measurement error in calcium intake from dietary instrument tools, and the possibility of residual confounding factors.

In summary, our study provides evidence suggesting that calcium intake is inversely associated with the prevalence of colorectal adenoma in Korean populations.

\section{ACKNOWLEDGMENTS}

This study was supported by Basic Science Research Program through the National Research Foundation of Korea, which is funded by the Korean Government (NRF-2011-0011028).

\section{CONFLICTS OF INTEREST}

No potential conflicts of interest were disclosed.

\section{REFERENCES}

1. Ferlay J, Soerjomataram I, Ervik M, Dikshit R, Eser S, Mathers C, et al. GLOBOCAN 2012 v1.0, Cancer Incidence and Mortality Worldwide: IARC CancerBase No. 11 [Internet]. Lyon, France: International Agency for Research on Cancer; 2013. Available from: http://globocan.iarc.fr. Accessed May 31, 2015.

2. Jung KW, Won YJ, Kong HJ, Oh CM, Cho H, Lee DH, et al. Cancer statistics in Korea: incidence, mortality, survival, and prevalence in 2012. Cancer Res Treat 2015:47:127-41.

3. Vogelstein B, Fearon ER, Hamilton SR, Kern SE, Preisinger AC, Leppert $\mathrm{M}$, et al. Genetic alterations during colorectal-tumor development. N Engl J Med 1988;319:525-32.

4. Keum N, Lee DH, Greenwood DC, Zhang X, Giovannucci EL. Calcium intake and colorectal adenoma risk: dose-response meta-analysis of prospective observational studies. Int J Cancer 2015;136:1680-7.

5. Huncharek M, Muscat J, Kupelnick B. Colorectal cancer risk and dietary intake of calcium, vitamin $\mathrm{D}$, and dairy products: a metaanalysis of 26,335 cases from 60 observational studies. Nutr Cancer 2009;61:47-69.

6. Wactawski-Wende J, Kotchen JM, Anderson GL, Assaf AR, Brunner RL, O'Sullivan MJ, et al; Women's Health Initiative Investigators. Calcium plus vitamin D supplementation and the risk of colorectal cancer. N Engl J Med 2006;354:684-96.

7. Lamprecht SA, Lipkin M. Chemoprevention of colon cancer by calcium, vitamin D and folate: molecular mechanisms. Nat Rev Cancer 2003;3:601-14.

8. Yamaji T, Iwasaki M, Sasazuki S, Sakamoto H, Yoshida T, Tsugane S. Association between plasma 25 -hydroxyvitamin D and colorectal adenoma according to dietary calcium intake and vitamin D receptor polymorphism. Am J Epidemiol 2012;175:236-44.

9. Ishihara J, Inoue M, Iwasaki M, Sasazuki S, Tsugane S. Dietary calcium, vitamin D, and the risk of colorectal cancer. Am J Clin Nutr 2008;88:1576-83. 
10. Shin A, Li H, Shu XO, Yang G, Gao YT, Zheng W. Dietary intake of calcium, fiber and other micronutrients in relation to colorectal cancer risk: results from the Shanghai Women's Health Study. Int J Cancer 2006;119:2938-42.

11. Ahn Y, Kwon E, Shim JE, Park MK, Joo Y, Kimm K, et al. Validation and reproducibility of food frequency questionnaire for Korean genome epidemiologic study. Eur J Clin Nutr 2007;61:1435-41.

12. Willett W, Stampfer MJ. Total energy intake: implications for epidemiologic analyses. Am J Epidemiol 1986;124:17-27.

13. Baron JA, Beach M, Mandel JS, van Stolk RU, Haile RW, Sandler $\mathrm{RS}$, et al. Calcium supplements for the prevention of colorectal adenomas. Calcium Polyp Prevention Study Group. N Engl J Med 1999:340:101-7.

14. Korea Centers for Disease Control and Prevention. 2013 Korean
National Health Statistics - KNHANES. Cheongju, Korea Center for Disease Control and Prevention, 2013.

15. Newmark HL, Wargovich MJ, Bruce WR. Colon cancer and dietary fat, phosphate, and calcium: a hypothesis. J Natl Cancer Inst 1984:72:1323-5.

16. Aggarwal A, Prinz-Wohlgenannt M, Tennakoon S, Höbaus J, Boudot C, Mentaverri R, et al. The calcium-sensing receptor: A promising target for prevention of colorectal cancer. [published online ahead of print February 18, 2015]. Biochim Biophys Acta. doi: 10.1016/j.bbamcr. 2015.02.011.

17. Kállay E, Bajna E, Wrba F, Kriwanek S, Peterlik M, Cross HS. Dietary calcium and growth modulation of human colon cancer cells: role of the extracellular calcium-sensing receptor. Cancer Detect Prev 2000;24:127-36. 